\title{
3 Qualitätsmanagement: Formale und rechtliche Vorgaben
}

Julia Bellabarba

Dieses Kapitel erklärt die gesetzlichen und normativen Vorgaben, die für das Qualitätsmanagement aller ärztlichen, psychotherapeutischen und kinderpsychotherapeutischen Praxen und MVZ gelten, soweit sie im Rahmen der GKV Leistungen erbringen und abrechnen.

Qualitätsmanagement in der ambulanten Versorgung der Patienten ist für niedergelassene Ärzte und Psychologen zur Verpflichtung geworden. Relevant sind dabei die Vorgaben des SGB V $\mathbb{1 3 6}$ a und die Richtlinien des Gemeinsamen Bundesausschusses. Diese gesetzlichen Vorgaben werden im Folgenden erläutert. Sie gelten für:

- niedergelassene Ärzte aller Fachrichtungen

- niedergelassene psychologische Psychotherapeuten (für Erwachsene, Kinder und Jugendliche)

- Medizinische Versorgungszentren

Zum 01.01.2004 wurde das fünfte Sozialgesetzbuches (Gesetz zur Modernisierung der Gesetzlichen Krankenversicherung) geändert. Es enthält in $\$ 135$ a (2) folgende Regelung: „Vertragsärzte, medizinische Versorgungszentren, zugelassene Krankenhäuser (...) sind (...) verpflichtet, (...) einrichtungsintern ein Qualitätsmanagement einzuführen und weiterzuentwickeln."

Der Gesetzgeber hat nicht festgelegt, in welcher Form und bis zu welchem Zeitpunkt ein „einrichtungsinternes Qualitätsmanagement“ eingeführt werden soll. Dies festzulegen obliegt dem Gemeinsamen Bundesausschuss: GBA $\$ 136 \mathrm{a}(1)$,Der Gemeinsame Bundesausschuss bestimmt für die vertragsärztliche Versorgung durch Richtlinien (...) die verpflichtenden Maßnahmen der 
Qualitätssicherung nach $\$ 135$ a Abs. 2 sowie die grundsätzlichen Anforderungen an ein einrichtungsinternes Qualitätsmanagement (...). “ Die gesetzliche Verpflichtung zum praxisinternen Qualitätsmanagement, die im Januar 2004 in Kraft getreten ist, wurde in der QM-Richtlinie des Gemeinsamen Bundesausschusses vom 18.10.2005 erläutert. Sie finden eine verkürzte Version der QM-Richtlinie des GBA in Anhang 4, mit Hinweisen der Autorinnen, in welchem Kapitel dieses Buches praktische Ideen zur Umsetzung der Richtlinie stehen. Die Richtlinie steht vollständig unter: www.g-ba.de/informationen/ richtlinien/18/.

\section{Wichtige Eckdaten der Richtlinie sind}

Keine Festlegung auf ein bestimmtes QM-System

Keine Verpflichtung zur Praxis-Zertifizierung

Keine Verpflichtung, Schulungen zum Thema zu besuchen

Die Praxen müssen QM spätestens ab 1.1.2008 umsetzen.

Die Überprüfung der Umsetzung der Richtlinie obliegt der KV.

Die wichtigsten Anforderungen an das Qualitätsmanagement einer Praxis sind in der QM-Richtlinie wie folgt beschrieben:

\section{Patientenversorgung}

- Ausrichtung der Versorgung an medizinischen Standards und Leitlinien

- Patientensicherheit, Patientenmitwirkung, Patienteninformation und Beratung

- Strukturierung von Behandlungsabläufen

- Notfallmanagement

\section{Praxisführung/Mitarbeiter/Organisation}

- Regelung von Verantwortlichkeiten

- Mitarbeiterorientierung (z.B. Arbeitsschutz, Fort- und Weiterbildung)

- Praxismanagement (z.B. Terminplanung, Datenschutz, Hygiene)

- Gestaltung von Kommunikationsprozessen (intern/extern) und Informationsmanagement

- Integration bestehender QS-Maßnahmen ins QM-System

Folgende Instrumente sind dabei zu nutzen

- Festlegung von konkreten Qualitätszielen für die Praxis und nachweisbare Überprüfung derselben sowie Dokumentation

- regelmäßige strukturierte Teambesprechungen 
- Prozessbeschreibungen

- Dokumentation von Behandlungsverläufen und Beratungen

- Patientenbefragungen, wenn möglich mit validen Fragebögen

- Beschwerdemanagement und Fehlermanagement

- Erkennen und Nutzen von Fehlern und Beinahefehlern zur Einleitung von Verbesserungsprozessen

- Organigramm

Somit bildet die QM-Richtlinie des Gemeinsamen Bundesauschusses drei relevante Trends in der vertragsärztlichen und vertragspsychotherapeutischen Versorgung ab:

- die Ausrichtung der ambulanten Versorgung an Leitlinien und evidenzbasierter Medizin

- das Postulat, den Patienten als mündigen Partner zu behandeln

- die offensive Thematisierung von Patientensicherheit/Fehlermanagement in der Arztpraxis

2010 werden die Umsetzung und der Nutzen praxisinternen QMs durch den GBA evaluiert. Nach 2010 erfolgt eine Entscheidung, ob die Richtlinie gewinnbringend umgesetzt wurde und in welcher Form Praxen, die kein QM umgesetzt haben, sanktioniert werden sollen. Die von der Gesundheitspolitik und von der Gesetzgebung gewollte Verpflichtung zum einrichtungsinternen QM bedeutet, dass die hohe Qualität der medizinischen Versorgung auch im ambulanten Bereich nicht mehr dem Zufall oder dem individuellen Bestreben der Praxisinhaber überlassen wird, sondern dass verbindlich festgelegt wird, auf welchen Ebenen und mit welchen Werkzeugen die Qualität eines so komplexen „Produktes“ wie die ambulante medizinische/psychotherapeutische Patientenversorgung organisiert werden soll. Diese Vorgabe bedeutet nicht, dass eine qualitativ hochwertige Versorgung bisher nicht existiert hat oder dass Praxen bisher keine wirksamen QM-Instrumente eingesetzt hätten. Vielmehr trägt die Richtlinie der Komplexität eines praxisinternen Qualitätsmanagement Rechnung und beschreibt sowohl die Inhalte, als auch die Instrumente, damit QM von allen Praxen systematisch und umfassend bearbeitet umgesetzt werden kann.

Aus den oben genannten Anforderungen an das Qualitätsmanagement einer Praxis geht hervor, dass medizinisches Qualitätsmanagement immer auf der Grundlage bestehender Gesetze, Rechtsnormen und Verordnungen, deren Anwendung in der vertragsärztlichen Versorgung schon lange verpflichtend ist, beruht. Und natürlich dient das Qualitätsmanagement einer medizinischen Organisation nicht zuletzt der effektiven Umsetzung sämtlicher geltender rechtlicher Normen und somit der Absicherung der Organisation in Hinblick auf haftungsrechtliche und strafrechtliche Auseinandersetzungen (sowohl mit Patienten und Angehörigen als auch mit Mitarbeitern und Auszubildenden). 
„Unter den Stichworten „Verrechtlichung der Medizin“, „Forensisches Risiko“ und „Defensive Medizin“lässt sich ein fortwirkender, für alle Beteiligten äußerst unguter circulus vitiosus darstellen. Diesen gilt es zu durchbrechen, was im Wesentlichen nur vermittels der Gewährleistung durchgängig möglichst positiver Ergebnisqualität auf der Grundlage adäquater Struktur- und Prozessqualität gelingen kann.“ (Bock 2009 S. 4)

Als einen ersten Schritt in Richtung Risikomanagement sollten Praxisinhaber prüfen, ob folgende Gesetze in der Praxis vorliegen, bekannt sind und umgesetzt werden (s. Tab. 2):

Tab. 2 Gesetze in der Praxis

\begin{tabular}{ll} 
Aushangpflichtige Gesetze und Verordnungen: & $\begin{array}{l}\text { Aushang- und unterweisungspflichtige Gesetze } \\
\text { und Verordnungen: }\end{array}$ \\
\hline Jugendarbeitschutzgesetz & Arbeitsschutzgesetz \\
Jugendarbeitschutzuntersuchung & Gefahrengutbeauftragtenverordnung \\
Mutterschutz & Unfallverhütungsvorschriften \\
Gesetzliche Unfallversicherung SGB VI & Biostoffverordnung \\
Arzthaftung & Brandschutz \\
Allgemeines Gleichbehandlungsgesetz & Datenschutz \\
Arbeitszeitgesetz & Erste Hilfe \\
Arbeitsgerichtgesetz & Gefahrenstoffe \\
& Heben und Tragen \\
& Leitern und Tritte \\
& Medizinproduktebetreiberverordnung \\
& Personenschutzanzügeverordnung \\
& Betriebsstättenverordnung \\
\hline
\end{tabular}

Darüber hinaus sollte jede Praxis prüfen, welche fachgruppenspezifischen Gesetze und Verordnungen für ihren Bereich aushangs- und unterweisungspflichtig sind und in welcher Form diese umgesetzt werden müssen (zum Beispiel Biostoffverordnung, Transfusionsgesetz, Gefahrenstoffverordnung, Strahlenschutzgesetz, etc.) (s. Tab. 3).

Tab. 3 Checkliste zur Umsetzung von Gesetzen

\begin{tabular}{l|l}
\hline Normen und Gesetze: Checkliste für Praxen & $\checkmark$ \\
\hline Welche Gesetze, Verordnungen und Normen sind für unsere Praxis relevant? & \\
\hline Wie organisieren wir die Unterweisungspflicht? & \\
\hline Wie organisieren wir die Aushangpflicht? & \\
Möglich wäre ein Ordner, auch in digitaler Form, zu dem das Praxispersonal Zugang hat. & \\
\hline Wer ist für die Einhaltung und Aktualisierung zuständig? &
\end{tabular}




\section{Literatur}

Berg D, Ulsenheimer K (Hrsg.): Patientensicherheit, Arzthaftung, Praxis- und Krankenhausorganisation. Berlin: Springer 2006

Bock R-W: Recht für Krankenhaus und Arztpraxis. Berlin: Medizinisch Wissenschaftliche Verlagsgesellschaft 2009

Holzer E, Thomeczek C, Hauke E, Conen D, Hochreutener MA: Patientensicherheit. Leitfaden für den Umgang mit Risiken im Gesundheitswesen. Wien: Facultas 2005

Plank J, Hein T (Hrsg.): Rechtssichere Erfüllung der gesetzlichen Qualitäts- und Sicherheitsvorschriften im Gesundheitswesen. Merching: Forum 2006

Rieger HJ (Hrsg.): Lexikon des Arztrechts. Heidelberg: Springer 2003

Sens B: Risiko- und Qualitätsmanagement - ein Widerspruch? Gesundh.ökon Qual.manag 2004; 9: 291-92

\section{Links}

www.g-ba.de

www.kbv.de/themen/6102.html

www.bgw-online.de

www.kbv.de/qep/11625.html

www.kvb.de/servlet/PB/show/1116432/QM-Richtlinienbroschuere_4_2008.pdf 